\title{
PREDICTION OF THERMAL ERRORS IN MACHINE TOOLS THROUGH DECOUPLED SIMULATIONS USING GENETIC ALGORITHM AND ARTIFICIAL NEURAL NETWORKS
}

\author{
T. Suresh Kumar ${ }^{1 *}$, J. Glänzel ${ }^{1}$, M. Bergmann ${ }^{1}$, M. Putz ${ }^{1}$ \\ ${ }^{1}$ Fraunhofer Institute for Machine Tools and Forming Technology IWU, Chemnitz, Germany \\ *Corresponding author; e-mail: Tharun.Suresh.Kumar@iwu.fraunhofer.de
}

\begin{abstract}
Thermal errors are one of the major contributors towards positioning discrepancies in machine tools in precision machining. Along with friction and waste heat generated from production processes and internal heat sources, environmental influences around the machine tool create considerable thermal gradients followed by non-linear structural deformations. Efficient quantification of these three contributing sources of thermal errors are required in order to formulate a reliable thermal-error compensation system. The creation of all possible thermal configurations, which a machine tool could be subjected to, is experimentally infeasible and requires complex and time-consuming coupled flow and thermo-structural simulations. This paper presents a new approach in thermal error prediction by using CFD and finite element (FE) simulations to train a three-level interconnected neural network system. The first level essentially decouples flow simulations from thermo-structural simulations using optimal FE node points found using a Genetic Algorithm (GA), which significantly reduces the required training data. The boundary convection data obtained from this level is used in the second level to predict possible thermal configurations of the machine tool, after careful consideration of parameters related to internal heat sources and production processes. The third level maps these thermal configurations onto displacements on the machine tool.
\end{abstract}

\section{Keywords:}

Flow-thermal-structural simulations, FE-Simulation, Genetic Algorithm, Artificial Neural Networks

\section{INTRODUCTION}

The three main sources of thermal errors in machine tools involve the actual machining/production process, internal heat sources caused mainly through heat dissipation or power loss from components and finally, environmental influences. These three error sources along with other smaller sources contribute to around $70 \%$ of the total machining errors [Bryan 1990]. Even though, all the three main sources are considered equally important to thermal errors [Bräunig 2018], environmental influences are neglected by most researchers/manufactures due to the experimental and numerical difficulties involved in measuring and testing them. Environmental testing on machine tools requires specialized climate chambers and a wide range of metrological equipment, which makes experimental investigation infeasible for most researchers. Numerical investigation in the form of Computational Fluid Dynamics (CFD) simulations can be used to effectively estimate the thermal behaviour between the machine tool and its surrounding air [Glänzel 2016]. For the study of forced convection, environmental parameters such as air inlet temperature, velocity and flow directions can be used as input variables and heat transfer coefficients (HTCs) can be obtained along the machine-air interface using CFD simulations. These HTCs serve as boundary convection data for structural simulations. After definition of other boundary conditions which define internal heat sources and production processes, thermo-structural (finite element method - FEM) simulations can be used to compute the tool centre point (TCP) displacements of the machine tool. This two-step coupled (CFD-FEM) simulation procedure is extremely time-consuming when new boundary conditions have to be investigated or complex geometries are involved. The fluid, thermal and structural simulations have to re-run again based on the changes made to the simulation model.

Decoupling of CFD simulations from thermo-structural simulations have been successfully performed using Characteristic Diagrams (CDs) in [Glänzel 2016]. CDs are one of the tools most commonly used by engineers in order to approximate real valued functions that depend on one or more input variables. The CDs discussed in this paper are based on smoothed grid regression technique as suggested in [Naumann 2012] and have been improved to high dimensional CDs which are able to approximate thermo-elastic deformations in machine tools. CDs map a 
set of input variables onto a single output variable continuously. They consist of a grid of support points along with kernel functions (1D or 2D) which describe the interpolation in between, refer [Naumann 2016].

Using data obtained from a limited number of CFD simulations run in advance, the CDs are trained for different combinations of environmental parameters (e.g. air temperature, air flow velocity, flow directions). They map the corresponding HTCs at the positions of FE-nodes at the interface between machine tool surface and surrounding air. The trained CDs are then used to predict HTCs for any environmental load case. However, for complex geometries and moving components, extensive training data is required. This data volume can be significantly reduced using a clustering technique which involves Genetic Algorithm (GA) [Kumar 2019]. This approach finds optimal subsets of FE node points at the machine-air-interface, for which CDs are formulated and trained. Thereby, the training process/data is reduced to a selected number of FE-node points. This approach was validated with a coupled simulation system in [Kumar 2019]. The results were encouraging with deviations in temperature and total mesh displacement at a selected point representing the TCP (tool centre point) by values of $0.5 \%$ and $4 \%$ respectively. The decoupling approach was experimentally validated in [Kumar 2020]. Temperature and displacement measurements were performed in the climate chamber of the Fraunhofer Institute for Machine Tools and Forming Technology (IWU) in Chemnitz and used to validate the simulations. In the best set of results, the difference in temperature and displacement reading at different sensor positions were found to be $+/-1^{\circ} \mathrm{C}$ and $+/-7 \mu \mathrm{m}$ respectively.

This paper attempts to further enhance the thermal error prediction method by replacing the CDs with Artificial Neural Networks (ANNs). ANNs have been adopted by serval researchers all around world for thermal error compensation in machine tools. Among the existing learning algorithms, neural networks are proposedly best suited when highly non-linear input-output relationships are involved. ANNs more efficiently handle huge amounts of training data and variables. Being an inherently parallel architecture, the updating and prediction processes are expected to be faster. However, most of the studies address the problem only partially. For example, in [Jian 2019] regression neural networks are used to predict thermal displacements in CNC-lathe spindle for variations in spindle speeds. [Wang 2019] proposed a method to compensate thermal error in $\mathrm{CNC}$ grinding machines using a hybrid convolutional neural network system in order to mapping the temperature change against the thermal response of the machine tool. In an earlier research by [Chen 1996], they used both a multiple regression analysis (MRA) model and an artificial neural network (ANN) model for thermal error compensation of a horizontal machining centre. In all the above mentioned and similar researches, it could be observed that the methods were tested for very limited error sources, operating conditions or for any particular direction of spindle feed. Being dependent on experimental data for training, they were proven effective within the training data range, but were expected to fail to extrapolate to unknown conditions. In a recent research [Santos 2018], FEM simulations are used to train neural networks to predict thermal errors in a five-axis CNC machine tool. However, environmental influences (forced convection and HTC contours) which play a major role (upto $40 \%$ in large machines [Bräunig 2018]) in thermal errors were only approximated using analytical calculations in the FE-model. When many papers show that a reduction of the thermal error to about $10 \%$ is possible, others state that this is only possible when the ANN is applied to the training data and that on independent data sets the reduction is to about $20 \%$. This proves that,the training data has to cover the whole expected range of the operating conditions. However, the process of obtaining such data can take several days for internal heating tests and several weeks or more for the environmental tests [Longstaff 2003].

In this paper, a three-level interconnected neural network structure (section 4) is introduced which is expected to overcome the above mentioned shortcomings in training of the neural networks. Being completely CFD-FE simulation based, any environmental, internal and production sources of thermal errors can be effectively incorporated and used for training the ANNs. For example, a machine operating in Germany (cold and dry) has completely different environmental conditions as compared to in Vietnam (hot and humid). The factory measurement based training data obtained from Germany could prove ineffective in Vietnam. The simulation based training can mitigate this problem by instantaneously performing CFD simulations and adding them to the online ANN system for new thermal configurations on the machine tool.

Nueral Network- optimal node 1

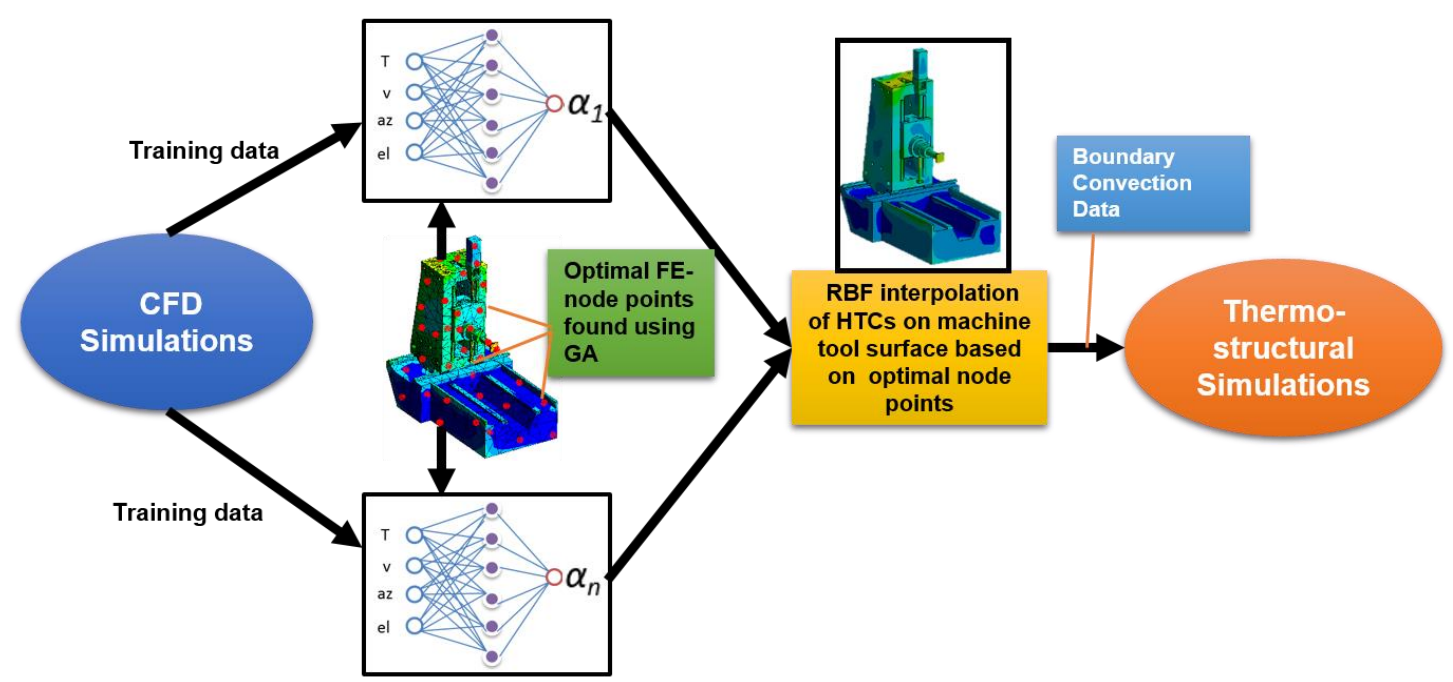

Nueral Network- optimal node $\mathrm{n}$

Fig. 1: Decoupling of CFD-Thermo-structural simulations

MM Science Journal | 2021 | Special Issue on ICTIMT2021 


\section{ANN-BASED DECOUPLING}

This section discusses the fundamental process structure of the decoupling approach using ANNs. This process is an intermediate step in the creation of the 3-level ANN structure described in section 4. ANN-based decoupling of fluid and thermo-structural simulations is similar in approach to the decoupling using CDs ([Glänzel 2016] and [Kumar 2019]).

Fig. 1 shows the decoupling approach using a series of similar neural networks trained using CFD simulations. A CFD simulation model of the machine tool geometry within a fluid domain is set up and HTC values are exported as CSV-files (comma separated values) at the machine-air interface under varying environmental load cases. These load cases, which are combinations of air flow temperature, velocity and flow directions, represent the different ambient conditions a particular machine tool can be subjected to. The mapping between these load cases and the HTC values obtained at the machine-air interfaces through CFD simulations serve as the training data for ANNs.

To reduce the data needed to train the ANNs, a clustering technique is developed. It incorporates optimal subset search of surface FE nodes using an optimization technique (genetic algorithm-GA) and subsequent interpolation of HTC values using radial basis functions (RBF). Both operations are performed using MATLAB scripts. As shown in Fig. 1, GA finds the best set of FE node points on the machine surface which gives the least interpolation error between the CFD-simulated and RBF-interpolated HTCs for each face under any particular load case. The training data for ANNs are based only on these optimal subsets, whereby its amount is reduced drastically. For illustration, in [Kumar 2019], 144 optimal node points were determined by GA from a machine-air interface consisting of approximately 100,000 node points which enabled a reduction in the number of FE-nodes which required training by $99.8 \%$.

Once trained, ANNs formulated at each optimal node point are capable of predicting HTC values for any user-defined load case. The predicted HTC values at the optimal subsets serve as RBF points to interpolate the HTCs at the machine-air interface, which is used as the boundary (convection) data for thermo-structural simulations.

The trained neural networks remove the dependency of thermo-structural simulations on CFD simulations for boundary convection data. Any thermal configuration and corresponding convection data can be attained at the optimal node points and interpolated over the entire machine-air interface. Thus, the decoupling of simulations is attained.

\section{OPTIMAL SUBSET SEARCH USING GENETIC ALGORITHM}

\subsection{Optimal Subset Problem}

Clustering is the process of grouping a set of objects in such a way that objects in the same group, called a cluster, are more similar (based on the objective) to each other than to those in other groups. Many algorithms have been developed to tackle clustering problems in a variety of application domains, including the hierarchica agglomerative CA, k-means, and self-organizing maps. The most popular algorithms are probably the fuzzy c-means and the k-means algorithms. All of these algorithms rely on Euclidean distances from cluster centroids as the criterion function. Therefore, they are limited to detecting spherical clusters and do not work well with non-Gaussian data. In simple words, the solution gets confined to local minima.

The search for a universal or at least more generic search algorithm led to the discussion on GAs. The GA attempts to find the most optimal solution to the problem by genetically breeding a population of individuals over a series of generations and effectively overcomes local minima on basis of the 'Darwinian principle of reproduction and survival of the fittest' in analogy to naturally occurring genetic operations such as crossover and mutation (refer [Koza 1995] and [Koenig 2001]).

As discussed in the previous section, the purpose of clustering in the decoupling approach is the reduction in the number of FE nodes and corresponding HTC values used for training neural networks. Maintaining accuracy in interpolation even after reduction of nodes is important. This is attained by finding optimal subsets of FE-nodes on the machine tool surface, which will be used to build an RBF-based interpolation function. The GA addresses the 'Optimal Subset Problem' [Glänzel 2016] by minimizing the weighting function $f$ as in Eq. 1 .

$\min _{S \subset V} f(S)$

$|S|=m$

In this context $V$ defines the whole set of nodes on the machine surface (machine-air interface), corresponding to the $N$ nodes $x_{1}, x_{2}, \ldots x_{N}$ of the finite element mesh. $f_{s}$ in Eq. 2. corresponds to the radial basis interpolation function [Glänzel 2016]. CFD-simulated HTC values are represented by $w_{1}, w_{2}, \ldots w_{N}$ corresponding to each node. In the decoupling approach a subset $S$ with cardinal number $m$ is selected from $V$ and the objective function $f$ calculates the interpolation error between CFD-simulated HTC values and GA-interpolated ones over an entire machine face. Eq. 2 formulates this point-wise, whereby $f(S)$ becomes zero if $m=N$ and becomes greater than zero otherwise.

$f(S)=\max _{i=1 \ldots \mathrm{N}}\left|f_{S}\left(x_{i}\right)-w_{i}\right|$

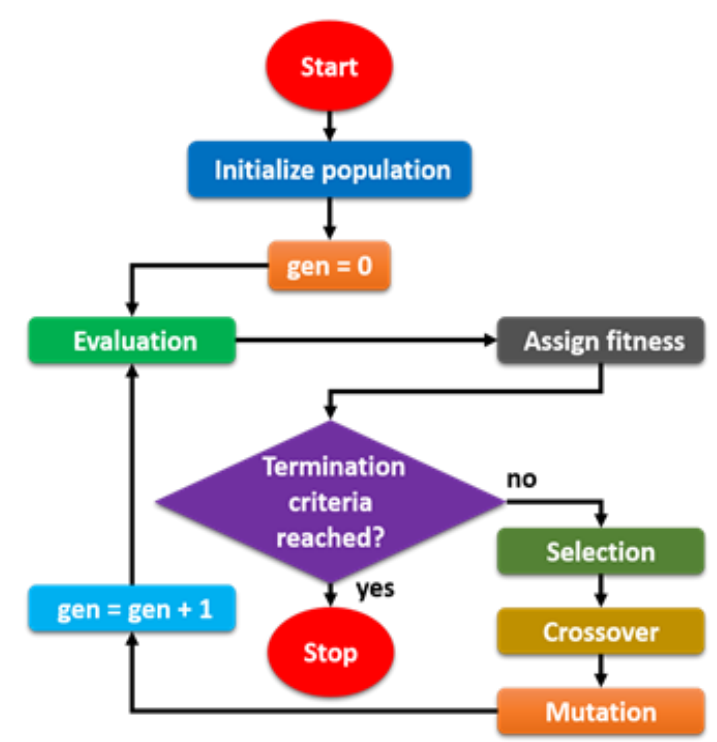

Fig. 2: GA working principle 


\subsection{Genetic Algorithm implementation}

The working principle of GA adopted for clustering is shown in Fig. 2. GA begins its search from a random initial population of solutions. For optimal subset search, the random FE node numbers (genes) on a particular machine surface face will constitute the population. A fitness value is assigned for each set (chromosomes) of FE node points based on the objective/fitness function (1), which finds the norm error between actual HTC values (obtained from simulation) and RBF interpolated HTC values using a particular chromosome. The chromosomes are sorted based on the lowest fitness values i.e. the least error. If the termination condition is satisfied, the GA process will stop. The termination condition could be a maximum number of generations or best (lowest) permissible fitness. If the termination criterion is not satisfied, then changes are made to the population using genetic operators - selection, crossover and mutation. In general, the exploitation of the accumulated information resulting from GA search is done by the selection and crossover mechanism [Koza 1995] while the exploration to new regions of the search space is accounted for by mutation as discussed in [Soni 2014].

\section{INTERCONNECTED ANN STRUCTURE}

\subsection{Principle of ANN for Thermal Error Prediction}

An ANN consists of two fundamental components - artificial neurons which process the input data and the network which connects these neurons [Abdulshahed 2013]. The architecture of an artificial neuron used in the implementation of the three-level network in this paper is shown in Fig. 3. The neuron receives a set of input variables $\left(\mathrm{x}_{\mathrm{i}}\right)$ and they are multiplied by a corresponding weight $\left(\mathrm{w}_{\mathrm{i}}\right)$. The weighted inputs are processed by the summation function (S) followed by an activation function [Nagata 2003], which transforms the summation into a more desired output. The summation function essentially performs the matrix multiplication of the weight vectors and the input values.

There are a lot of different activation functions used in neural networks. Here, a sigmoid function (Eq. 3), also known as the logistic function is used in the neural networks to transform outputs into a specified range, i.e. between 0 and 1.

$\sigma(S)=\frac{1}{1+e^{-S}}$

There are several types of neural networks based on the specific application. In this paper, multi-layer feed-forward neural networks are utilized to connect the neurons. In this network, the data is transferred in only the forward direction, from the input neurons, through hidden neurons and to the output neurons, as shown in Fig 4. Each neuron in one layer has direct connections to the neurons of the subsequent layer.

For training the network, the back-propagation (BP) algorithm, as described in [Rumelhart 1986] is adopted. BP-based ANN modelling has proved to be a suitable nonlinear modelling method in a number of research studies [Abdulshahed 2013]. Initially, random values between 0 and 1 are assigned to the weights $\left(\mathrm{w}_{\mathrm{i}}\right)$ [Nasr 2013]. The feedforward step starts by assigning data to the input layer. The input-output mapping information obtained from the sigmoid function at the inner neurons are transferred forward up to the output layer. Here, the output at each neuron (y) are compared with the desired data (D) and the

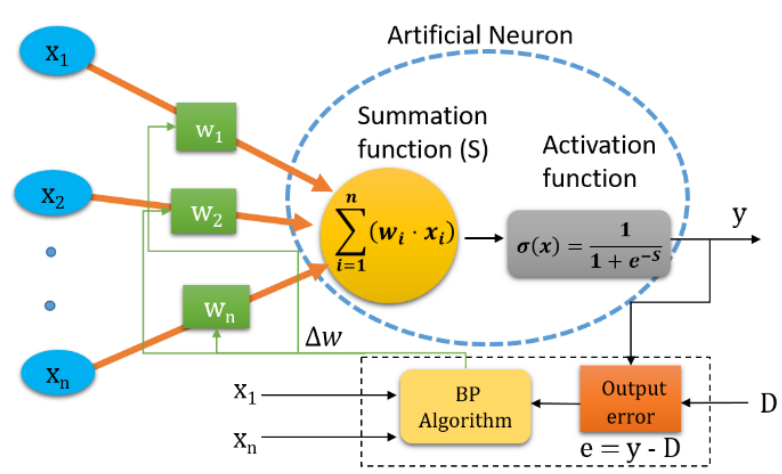

Fig. 3 : Artificial neuron architecture along with backpropagation algorithm (used in this paper)

resulting error (e) is used to adjust the corresponding weights, as shown in Fig. 3. This process is known as backpropagation. A feed-forward step along with a backpropagation comprises a cycle of training. The training ends when the error in the termination cycle reaches a specified threshold.

As mentioned in section 1, all three sources of thermal errors will be parametrized through a wide range of variables like temperature, air flow velocity, generated heat per volume, natural/forced convection, radiation etc. These features are on significantly different scales. Problems can arise in the neural network when the back-propagation assign weights based on a prominent variable instead of a sensible variable. Therefore, normalization of input variables is absolutely necessary. In this approach, 'MinMax' normalization is utilized to transform the input values into a decimal between 0 and 1 .

\subsection{Implementation of ANN Approach}

One of the most relevant advantages of ANNs in thermal research is the efficient handling of highly non-linear relationships in data. In several studies, it has been shown that thermal errors can be successfully predicted using ANNs [Abdulshahed 2013] [Guo 2010]. ANNs do not require detailed knowledge of the physical phenomena describing the system under analysis.

The idea being proposed in this paper is a three-level interconnected ANN based thermal error compensation system/module (Fig. 5), which is trained completely from simulations. The first level consists of a series of similar neural networks which are completely trained by CFD simulations. The methodology used to parameterize and estimate the environmental influences associated with the machine tool makes this ANN system unique. As mentioned in section 2 and 3 , optimal subsets of FE nodes are found on the machine tool surface using GA. Each optimal node point will have its own independent neural network. Therefore, the number of neural networks in the first level is equal to the number of optimal FE node points. Using

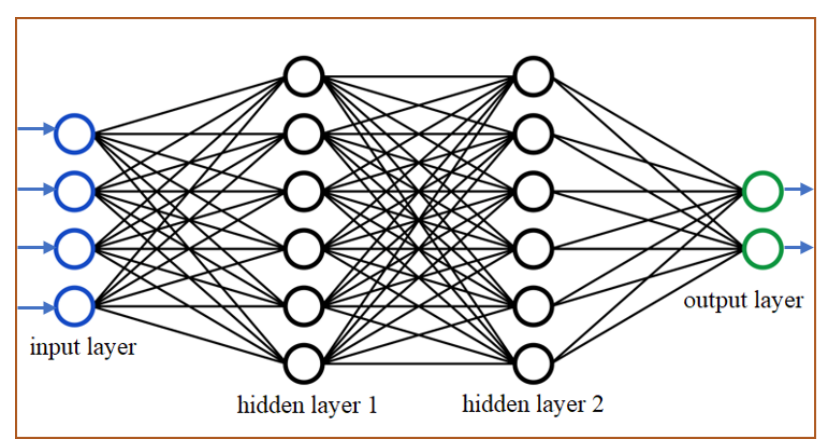

Fig. 4 : A four-layer feed forward ANN structure 


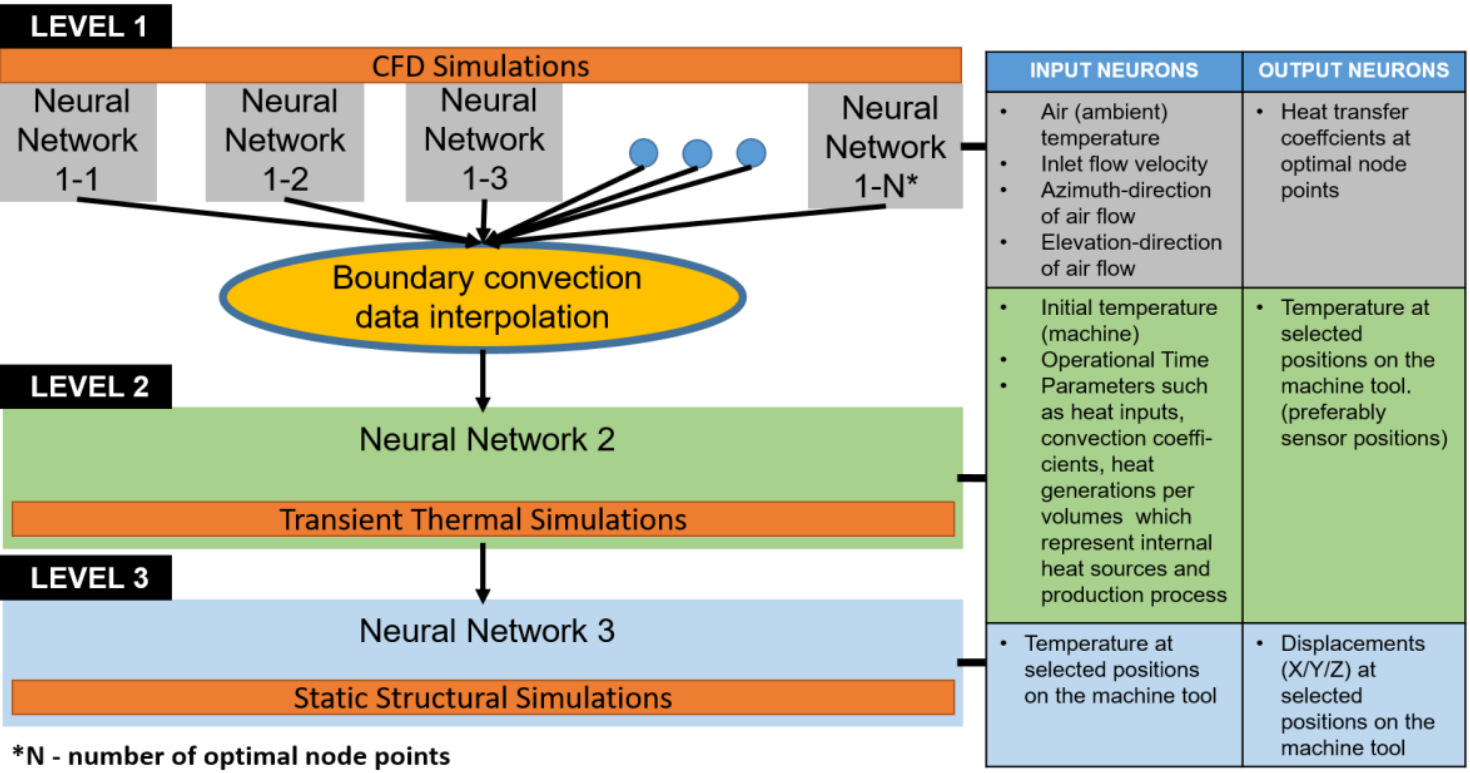

Fig. 5 : Three-level interconnected neural network structure

data from CFD simulations, environmental influences (ambient temperature, inlet flow velocity, air flow directions) are mapped onto HTC values only at these optimal nodes, whereby the amount of training data for neural networks is incredibly reduced without a significant loss of accuracy. The number of associated degrees of freedom is also reduced which makes the system more stable. After strategic training, each neural network predicts the HTC value for any environmental load case at the corresponding optimal node points. This predicted data can be interpolated over the complete machine tool surface, which is later used as the boundary convection data in thermo-structural simulations.

The second level consists of a single neural network, which is trained using transient thermal simulations. In this simulation model, the series of neural networks in the first level provide the boundary convection data, which represents the environmental influences on the machine tool. Along with this, other parameters which represent the internal heat sources (such as heat generated at various components, heat input on the surfaces etc.) and production processes (machining, influence of coolant, chips etc.) constitute the boundary parameters used in this thermal simulation model. Different thermal configurations possible on the machine tool are created using these simulations. Temperature probes are defined at the surface nodes which represent the temperature sensor positions. Therefore, as expected, the different boundary parameters are mapped onto certain temperature values in this neural network. The input and output neurons associated with this

level can be seen in Fig. 5. After training, any thermal configuration associated with the machine tool can be generated using the neural network system (level 2).

Once the different thermal configurations are simulated, the corresponding static displacements on the machine tool can be obtained. The third level which consists of a single neural network which maps these simulated temperature fields onto corresponding simulated displacements at specified positions on the machine tool. The third neural network is designed in such a way that it can also be trained using experimental readings obtained directly from the machine tool along with the simulations. The mapping obtained from this neural network will be utilized/incorporated by the NC-Program for the machine tool in thermal error compensation.

The most important prerequisites of simulation based ANN thermal error compensation system are the validated and accurate fluid and thermo-structural simulation models of the machine tool. Environmental testing for validation of fluid simulations is possible within the retrofitted climate chamber [Kumar 2020]. Similarly, the thermo-structural simulations can also be validated using the wide range of metrological equipment available at the institute.

\section{CASE STUDIES}

\subsection{Comparison of results with and without clustering}

Clustering of FE node points, in order to represent certain sections of the machine tool-air interface provides significant benefits irrespective of the training algorithm

HTC $\left[\mathbf{W m}^{-2} \mathrm{~K}^{-1}\right]$
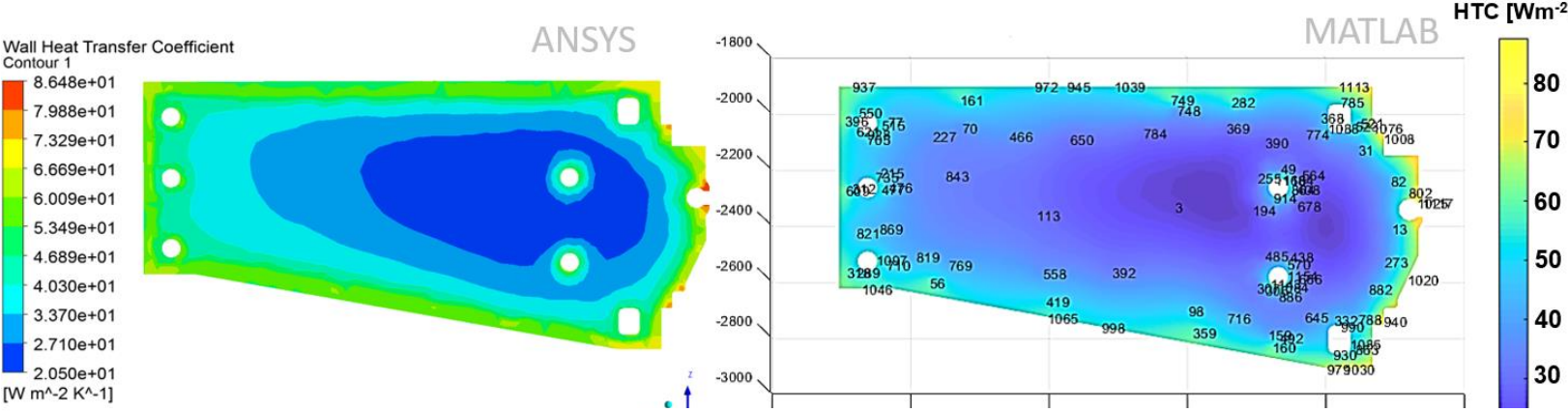

Fig. 6 : (a) HTC-plot from ANSYS-CFX (b) HTC-interpolated using 100 ONPs for same environmental boundary conditions

MM Science Journal | 2021 | Special Issue on ICTIMT2021 
Tab. 1 : Comparison- with and without ONPs

\begin{tabular}{c|c|c}
\hline Method & Without ONPs & With ONPs \\
\hline Mapping & $\begin{array}{l}(T,|v|, \tilde{x}, \tilde{y}, a z, e l) \\
\rightarrow \alpha(x, y)\end{array}$ & $\begin{array}{l}(T,|v|, a z, e l) \\
\rightarrow \alpha(O N P)\end{array}$ \\
$\begin{array}{c}\text { Number of } \\
\text { simulations } \\
\begin{array}{c}\text { Relative } \\
\text { Error in } \\
\text { HTCs }\end{array}\end{array}$ & $>160$ & 18 \\
\hline
\end{tabular}

(Characteristic Diagrams or Artificial Neural Networks). For a compensation system to be able to act/predict according to the real-life environmental/thermal behaviour of the machine tools, a huge amount of training data is required. A typical 5-axis machining unit involves numerous components and sub-components, dynamic parts and changing boundary conditions. A fine FE mesh is also required to effectively extract the heat transfer properties on the machine surface. Clustering, as explained in section 3, helps in extensively reducing the complexity of such models by finding optimal FE nodes, which effectively represents the convection behaviour of all the surface nodes.

For the current comparison, the mapping of environmental influences is considered. Without optimal node points, the mapping would have the structure as shown in Eq. 4. Seven variables (flow temperature, velocity, FE-node coordinates and flow directions-azimuth and elevation) have to be mapped onto the HTCs at each FE node point on the machine-air interface. The higher the number of variables involved, the less stable an approximation strategy becomes. To make it stable, more training data (load cases/ simulations) would be required, [Juba 2018].

$$
(T,|v|, \tilde{x}, \tilde{y}, \tilde{z}, a z, e l) \rightarrow \alpha(x, y, z)
$$

Optimal subsets solve this problem by modifying the mapping structure, Eq. 5. At each pre-determined and load independent optimal node point, the environmental influences are mapped onto HTCs. The reduction of variables improves the stability of the system and substantially reduces the amount of training data (simulations) required.

$$
(T,|v|, a z, e l) \rightarrow \alpha(O N P)
$$

For simple illustration, an inlet air flow facing 2D surface of a three-axis milling machine Auerbach ACW 630 is used. As tabulated in [Glänzel 2018], without optimal node points, more than 160 simulations were required to reduce the relative error for a test case to $11.4 \%$. For these simulations, six input variables were involved, as shown in Tab. 1. With 100 optimal node points, 18 simulations were enough to reduce the same relative error to $13.2 \%$. This tremendous reduction in number of simulations and training data without greatly compensating in accuracy shows the promising potential of this approach. The HTC plots obtained for an environmental test case from ANSYS-CFX and the one interpolated using 100 optimal node points (plotted in MATLAB) can be seen in Fig. 6 .

\subsection{Simulation-based validation of ANN-based correction method}

A complex and experimentally verifiable simulation model is used for the implementation of CD-based parameterization. The geometry chosen for this investigation is the 5-axis machining centre 'DMU 80' from

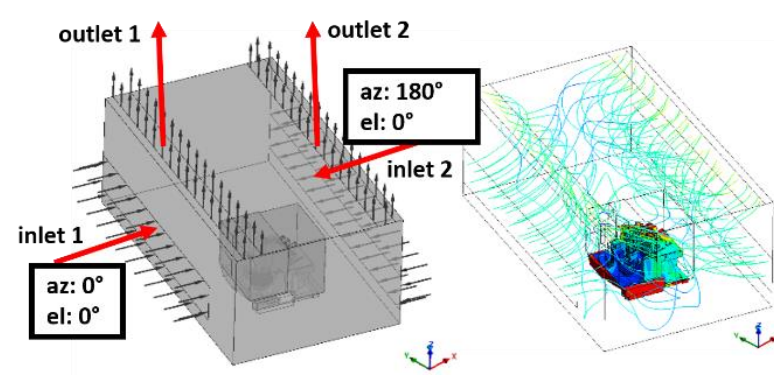

Fig. $7:$ (a) CFD-simulation model (b) HTC-contour and flow streamlines

DMG Mori. The machining centre comes with a housing which reduces the environmental impacts on the main machining region and other components. The CFD simulation model of the DMU 80 in the climate chamber can be seen in Fig. 7(a). The HTC contour obtained for a particular environmental load case along with the flow streamline can be seen in Fig. 7(b).

The CD-based decoupling approach, as mentioned in section 1, was already adopted [Kumar 2019] and experimentally validated [Kumar 2020] for a 3-axis machine tool, Auerbach ACW 630. The only training data used in these papers were CFD simulations in order to train the

Tab. 2 : Training load cases- Level 1

\begin{tabular}{c|c|c|c|c}
\hline $\begin{array}{c}\text { Load } \\
\text { Case }\end{array}$ & $\begin{array}{c}\mathbf{T}_{\mathrm{a}}-\text { Air } \\
\text { temperature } \\
\left({ }^{\circ} \mathbf{C}\right)\end{array}$ & $\begin{array}{c}\mathbf{V}_{\text {in }} \text { - Inlet } \\
\text { flow } \\
\text { velocity } \\
(\mathbf{m} / \mathbf{s})\end{array}$ & $\begin{array}{c}\text { az }- \\
\text { azimuth } \\
\text { angle of } \\
\text { inlet } \\
\text { flow }\left({ }^{\circ}\right)\end{array}$ & $\begin{array}{c}\text { el- } \\
\text { elevation } \\
\text { angle of } \\
\text { inlet flow } \\
\left({ }^{\circ}\right)\end{array}$ \\
\hline 1 & 20 & 1 & 0 & 0 \\
2 & 30 & 1 & 180 & 0 \\
3 & 20 & 5 & 180 & 0 \\
4 & 30 & 5 & 0 & 0 \\
\hline
\end{tabular}

Tab. 3 : Training load cases- Level 2

\begin{tabular}{c|c|c}
\hline $\begin{array}{c}\text { Analysis } \\
\text { Time (s) }\end{array}$ & $\begin{array}{c}\mathbf{T}_{\mathrm{i}} \text { - Initial temperature } \\
\text { of machine tool }\left({ }^{\circ} \mathbf{C}\right)\end{array}$ & $\begin{array}{c}\text { Heat generated } \\
\text { at Spindle } \\
\left(\mathbf{W} / \mathbf{m}^{3}\right)\end{array}$ \\
\hline 1000 & 60 & 10000 \\
1000 & 50 & 15000 \\
1000 & 50 & 10000 \\
1000 & 60 & 15000 \\
3000 & 60 & 10000 \\
3000 & 50 & 15000 \\
3000 & 50 & 10000 \\
3000 & 60 & 15000 \\
5000 & 60 & 10000 \\
5000 & 50 & 15000 \\
5000 & 50 & 15000 \\
5000 & 60 & \\
\hline
\end{tabular}


Tab. 4 : Test load cases

\begin{tabular}{c|c|c|c|c|c|c|c}
\hline Load Case & $\begin{array}{c}\mathbf{T}_{\mathrm{a}}-\text { Air } \\
\text { temperature } \\
\left({ }^{\circ} \mathbf{C}\right)\end{array}$ & $\begin{array}{c}\mathbf{V}_{\text {in }} \text { - Inlet flow } \\
\text { velocity } \\
(\mathbf{m} / \mathbf{s})\end{array}$ & $\begin{array}{c}\text { az - azimuth } \\
\text { angle of inlet } \\
\text { flow }\left({ }^{\circ}\right)\end{array}$ & $\begin{array}{c}\text { el- elevation } \\
\text { angle of inlet } \\
\text { flow }\left({ }^{\circ}\right)\end{array}$ & $\begin{array}{c}\text { Analysis } \\
\text { Time }(\mathbf{s})\end{array}$ & $\begin{array}{c}\mathbf{T}_{\mathrm{i}} \text { - initial } \\
\text { temperature } \\
\text { of machine } \\
\text { tool }\left({ }^{\circ} \mathbf{C}\right)\end{array}$ & $\begin{array}{c}\text { Heat } \\
\text { generated at } \\
\text { Spindle } \\
\left(\mathbf{W} / \mathbf{m}^{3}\right)\end{array}$ \\
\hline 1 & 23 & 3 & 0 & 0 & 1500 & 57 & 12000 \\
2 & 27 & 2 & 180 & 0 & 3900 & 53 & 14000 \\
\hline
\end{tabular}

CDs. Therefore, only the environmental influences as mentioned in section 2 were considered in these papers.

In this paper, the attempt is to create a holistic thermal error prediction system by gathering training data, not only from CFD simulations, but also from thermal and structural simulations, which represent internal heat sources and production processes. Instead of CDs, the three-leve interconnected neural network structure (Fig. 5) is utilized.

For the current investigation, the coupled fluid-thermalstructural simulations in ANSYS Workbench will be used to validate the ANN-based approach. Tab. 2 and 3 show the load cases used for training neural networks in Levels 1 and 2 respectively. The aim of these two levels are to predict any thermal configuration possible on the machine tool. In level 1, four load cases will be mapped onto HTCs at each ONP. The flow directions are varied with azimuth angles at $0^{\circ}$ and $180^{\circ}$ as shown in Fig. 7 (a). Further, transient thermal simulations will be carried out in order to train the neural network in level 2. Different combinations of analysis time, initial temperature of the machine and heat generated at the spindle are used to perform simulations under each boundary convection data obtained from ANNs in level 1. These thermal simulation parameters (Level 2) along with the environmental parameters (used to train in Level 1) are mapped onto the temperature values (temperature field) at specific points on the machine tool. This mapping serves as the training data for the ANN in level 2.

Once the different temperature fields are simulated, the corresponding static displacements can be obtained. Here specific points on the machine tool are selected to record the displacements in different directions. The mapping between temperature points and displacement serves as the training data for ANN in level 3.

For validation of ANN-based approach, two test load cases as shown in Tab. 4 are used. These are a combination of load parameters in levels 1 and 2, and they lie within the training boundaries. For comparison, certain points are selected on the machine tool as shown in Fig. 8. The temperature and displacement reading obtained at these points will be validated with those obtained from the threelevel ANN structure.

For the test load case in Table 4, the results as shown in Fig. 9 were obtained. At the selected temperature points,

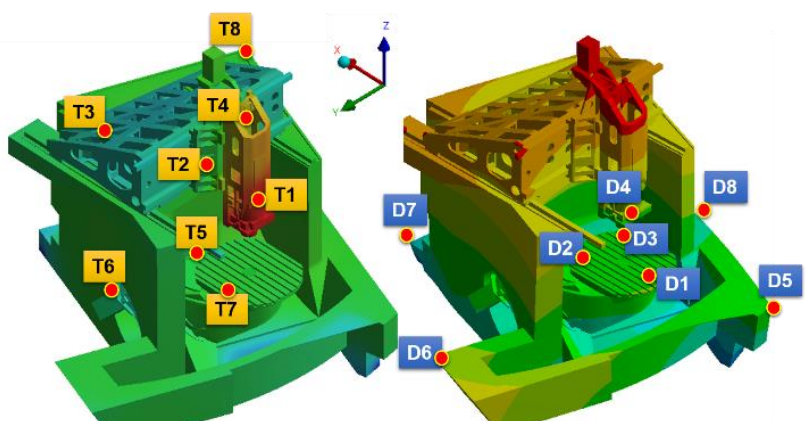

Fig. 8 (a) Temperature points and (b) Displacement points for validations
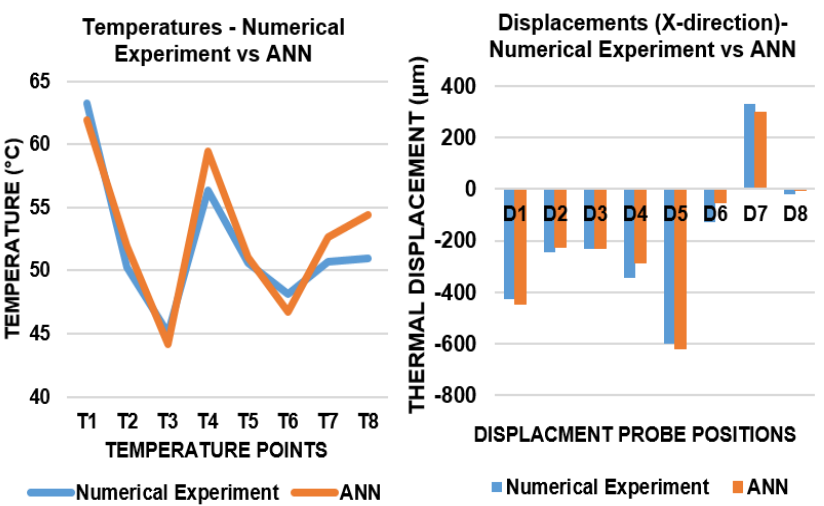

Fig. 9: Test Case 1 -Numerical Experiment vs ANN (a) temperature readings (b) displacement readings

the average of the relative error between the numerical experiment and the ANN-based approach was found to be a very small value of $3.4 \%$. On comparison between displacements in $\mathrm{X}, \mathrm{Y}$ and $\mathrm{Z}$ directions, the biggest deviations were observed in the $X$-direction. The average of the relative error at the displacement points was observed to be $21 \%$. The major contributors to this error are D6 and D8 which are extreme points on the machine bed. A better temperature field estimation in these regions could improve the displacement values. For this, better training of ANNs especially in level 1 is required. The average of errors in $Y$ and $Z$ directions were $15 \%$ and $19 \%$ respectively.

A similar trend was observed for test load case 2 as well. The average of relative error at the temperature points was $2.7 \%$. The displacements had average deviations of $20 \%$, $17 \%$ and $17 \%$ in $X, Y$ and $Z$ directions respectively (Fig. 10).

\section{CONCLUSION AND OUTLOOK}

This paper introduces a thermal error prediction method using a three-level interconnected neural network structure which can be completely trained by CFD and thermo-elastic simulations. The ANN system is prepared to operate in two modes- (i) addition of new simulations and prediction (ii)
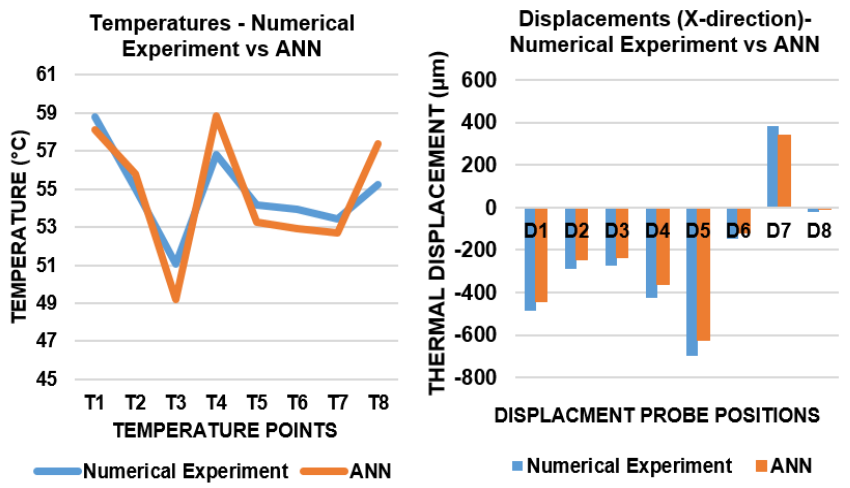

Fig. 10 : Test Case 2 -Numerical Experiment vs ANN (a) temperature readings (b) displacement readings 
prediction using existing data. The case studies mentioned in section 5 is performed in mode (ii)-prediction using existing data. Once a precise CFD-FE simulation model of the machine is established, any possible environmental, internal or production conditions on/in the machine tool can be instantly simulated and used for training. In comparison with existing thermal error correction systems, this method provides the flexibility to users to choose/add/update the training data based on any operating condition and removes the dependency on tedious and time-consuming experimental measurement data extraction for training purposes.

More simulations can potentially radically improve the prediction accuracy of this architecture. A comparison study with other prediction algorithms such as gradient boost or random forest is also planned in the near future. Paralle computing has already been implemented for fast extraction of simulation data [Kumar 2020], especially for ANN level 1. With ever-improving computational capabilities and reduced simulation models like model order reduction (MOR), this approach can be utilized as a holistic thermal error prediction system for the years to come. As a next step, the ANN method introduced in this paper will be validated experimentally in actual operating conditions of the machine tool, i.e. dry machining, with coolant and chips, and cool-down phases. After initial training, the ANNsystem can be incorporated as an error compensation module in any CNC operated machine tool. The training data can be updated online based on any new operational conditions of the machine tool through simulations.

\section{ACKNOWLEDGMENTS}

This research is being supported by the German Research Foundation in the context of the Collaborative Research Centers Transregio 96, subproject B09 (Project-ID 174223256 - TRR 96).

\section{REFERENCES}

[Bryan 1990] Bryan, J, International Status of Thermal Error Research. CIRP Annals Manufacturing Technology 39(2):645-656, 1990

[Bräunig 2018] Bräunig, M., Putz, M., Richter, C., Regel, J. Industrial consideration of thermal issues in machine tools. German Academic Society for Production Engineering (WGP) 2018

[Glänzel 2016] Glänzel J., Ihlenfeldt S., Naumann C., Putz M. Decoupling of fluid and thermo-elastic simulations on machine tools using characteristic diagrams, 10th CIRP Conference on Intelligent Computation in Manufacturing Engineering, CIRP ICME '16, Ischia, Italy, 2016.

[Naumann 2012] Naumann C., Priber U. Modellierung des Thermo-Elastischen Verhaltens von Werkzeugmaschinen mittels Hochdimensionaler Kennfelder. Proceedings Workshop Computational Intelligence, Dortmund, 2012.

[Naumann 2016] Naumann C , Putz M, Ihlenfeldt S, Kauschinger B, Theim X, Riedel M. Implementation And Demonstration Of Characteristic Diagram As Well As Structure Model Based Correction Of Thermo-Elastic Tool Center Point Displacements. Machines and manufacturing optimization, 2016
[Kumar 2019] Kumar TS., Glänzel J., Naumann C, Putz M. Parameterization of Environmental Influences by Automated Characteristic Diagrams for the Decoupled Fluid and Structural-Mechanical Simulations, Journal of Machine Engineering, Karpacz, 2019

[Kumar 2020] Kumar TS., Glänzel J., Tehel R., Putz M.Experimental Validation of Characteristic DiagramParameterization for Environment-Induced Thermal Interactions on Machine Tools in a Climate Chamber, 14th CIRP Conference on Intelligent Computation in Manufacturing Engineering, Gulf of Naples, Italy, 2020

[Juba 2018] Juba, B. and H. S. Le, Precision-Recall Versus Accuracy nd the Role of Large Data Sets, Association for the Advancement of Artificial Intelligence, 2018.

[Glänzel 218] Glänzel J., Naumann C., Ihlenfeldt S., Putz $M$. Efficient quantification of free and forced convection via the decoupling of thermo-mechanical and thermo-fluidic simulations of machine tools. Journal of Machine Engineering, Karpacz, 2018

[Rumelhart 1986] Rumelhart D. E., Hiton G. E., Williams R. J. Learning internal representations by error propagation. Parallel Distributed Processing, 1, pp. 318-362, MIT Press, Cambridge, MA, 1986.

[Abdulshahed 2013] Abdulshahed A., Longstaff A.P., Fletcher S., Mayers A. Comparative study of ANN and ANFIS prediction models for thermal error compensation on CNC machine tools. Laser Metrology and Machine PerformanceX, Buckinghamshire, 2013, pp.79-88.

[Nagata 2003] Nagata Y., Chu K.H. Optimization of a fermentation medium using neural networks and genetic algorithms. Biotechnology letters, vol. 25, pp. 1837-1842, 2003.

[Nasr 2013] Nasr N., Hafez H., Naggar H., Nakhla G. Application of artificial neural networks for modelling of biohydrogen production. International journal of hydrogen energy, vol. 38, pp. 3189-3195, 2013.

[Guo 2010] Guo Q., Yang J., Wu H. Application of ACOBPN to thermal error modelling of NC machine tool. The international journal of advanced manufacturing technology, vol. 50, pp. 667-675, 2010.

[Koza 1995] Koza JR. Survey of genetic algorithms and genetic programming. Proceedings of WESCON'95, 589594, 1995.

[Koenig 2001] Koenig AC. Study of mutation methods for Evolutionary Algorithms. CS 447-Advanced topics in Artificial Intelligence 2001

[Soni 2014] Soni N, Kumar T. Study of Various Mutation Operators in Genetic Algorithms. International Journal of Computer Science and Information Technologies, 5(3):4519-4521, 2014

[Jian 2019] Jian B, Wang C. Predicting spindle displacement caused by heat using the general regression neural network. The International Journal of Advanced Manufacturing Technology (2019) 104:4665-4674

[Wang 2018] Wang K, Shen H. Sensing and Compensating the Thermal Deformation of a Computer-numerical-control Grinding Machine Using a Hybrid Deep-learning Neural Network Scheme, Sensors and Materials, Vol. 31, No. 2 (2019) 399-409 
[Chen 1996] Chen J, Yuan J, Ni J. Thermal error modelling for real-time error compensation.Int $\mathrm{J}$ Adv Manuf Technol 1996;12:266-75.

[Santos 2018] Santos M, Batalha G. Numerical and experimental modeling of thermal errors in a five-axis CNC machining center, The International Journal of Advanced Manufacturing Technology (2018) 96:2619-2642

[Longstaff 2003] Longstaff A, Fletcher S, "Practical experience of thermal testing with reference to ISO 230 Part 3," in Laser metrology and machine performance $\mathrm{VI}$, Southampton, 2003, pp. 473-483.

[Kumar 2020] Kumar TS., Naumann A, Glänzel J., Putz M.

Parallel computing in automation of decoupled fluidthermostructural simulation approach, Journal of Machine Engineering 20(2):39-52, Karpacz, 2019 